\title{
Large-Biot-number non-isothermal flow of a thin film on a stationary or rotating cylinder
}

\author{
B. R. Duffy a and S. K. Wilson ${ }^{\mathrm{b}}$ \\ Department of Mathematics, University of Strathclyde, \\ Livingstone Tower, 26 Richmond Street, Glasgow G1 1XH
}

\begin{abstract}
Using the lubrication approximation we investigate two-dimensional steady flow of a thin film of fluid with temperature-dependent viscosity on a uniformly heated or cooled horizontal cylinder, which may be stationary or rotating about its axis, in the case when the Biot number (a measure of heat transfer at the free surface) is large. We show that the film thickness (but not the fluid velocity) may be obtained from that in the isothermal case by a simple re-scaling.
\end{abstract}

\section{Introduction}

The behaviour of a thin film of fluid on a cylinder is a problem of great industrial importance, occurring in, for example, heat exchangers, lubrication and coating processes (see, for instance, the comprehensive volume on coating edited by Kistler and Schweizer [1]). Two related paradigm problems, namely flow of prescribed flux on a stationary cylinder, and flow of prescribed mass on a rotating cylinder, have been studied extensively (see, for instance, Nusselt [2,3], Moffatt [4], Johnson [5]). We consider both of these problems in the case when the cylinder is uniformly heated or cooled, and the viscosity of the fluid is temperature-dependent, in the case when the Biot number (a non-dimensional measure of heat transfer at the free surface) is large. Wilson and Duffy [6] review work on other thin-film flows of a fluid with temperature-dependent viscosity, in all of which the case of large Biot number is included.

\section{Problem formulation}

We consider two-dimensional steady flow of a film of Newtonian fluid of uniform density $\rho$ and temperature-dependent viscosity $\mu$ on a circular cylinder of radius $a$ with its axis horizontal, the cylinder being at a prescribed uniform temperature $T_{0}$, hotter or colder than the surrounding atmosphere, at the uniform temperature $T_{\infty}$. We consider both purely gravity-driven flow of prescribed volume flux on the outside of a stationary cylinder (which we will refer to as "Nusselt flow"; cf Nusselt [2,3]) and flow on a cylinder that is rotating about its axis at uniform angular speed $\Omega$ (which we will refer to as "Moffatt flow"; cf Moffatt [4]); in the latter case the fluid may be on the inside of the cylinder ("rimming flow") or the outside ("coating flow"). The situation is sketched in Fig. 1, drawn for the case of coating flow.

Referred to the polar coordinates $r, \theta$ shown in Fig. 1, we take the free surface of the fluid to be at $r=a+h$ for either Nusselt flow or coating flow, and at $r=a-h$ for rimming flow, the film thickness being denoted by $h=h(\theta)$.

\footnotetext{
a e-mail: b.r.duffy@strath.ac.uk

b e-mail: s.k.wilson@strath.ac.uk
} 


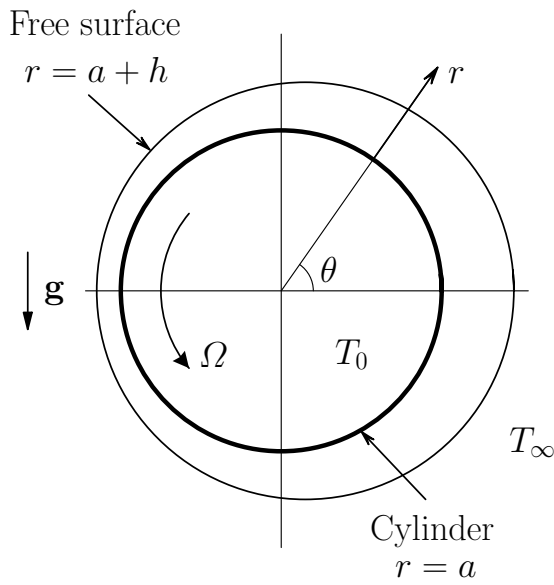

Fig. 1. Geometry of the problem: two-dimensional steady flow of a thin film of viscous fluid on a heated or cooled cylinder (drawn for the case of coating flow).

The fluid velocity $\mathbf{u}$, pressure $p$ and temperature $T$, all functions of $r$ and $\theta$, are governed by the familiar mass-conservation, Navier-Stokes and energy equations. On the cylinder $r=a$ the velocity $\mathbf{u}$ satisfies the no-slip and no-penetration conditions, and the temperature is $T=T_{0}$ (a prescribed constant). On the free surface $r=a \pm h$ the usual normal and tangential stress balances and the kinematic condition apply. Moreover, we shall take the Biot number (defined by $B=\epsilon a \alpha_{\mathrm{th}} / k_{\mathrm{th}}$, where the constants $k_{\mathrm{th}}$ and $\alpha_{\mathrm{th}}$ denote the thermal conductivity of the fluid and a surface heat-transfer coefficient) to be large, so that the free surface is at the same uniform temperature as the atmosphere, that is, $T=T_{\infty}$ on the free surface $r=a \pm h$. A large Biot number is certainly feasible physically; for example, for a film of water the parameter values $\epsilon=10^{-1}, a=0.05 \mathrm{~m}, k_{\mathrm{th}}=0.604 \mathrm{~kg} \mathrm{~m} \mathrm{~s}^{-3} \mathrm{~K}^{-1}$ and $\alpha_{\mathrm{th}}=10^{3} \mathrm{~W} \mathrm{~m}^{-2} \mathrm{~K}^{-1}$ lead to $B \simeq 10$.

The analysis is valid for an arbitrary dependence of viscosity on temperature, that is, for any viscosity function $\mu(T)$; without loss of generality we define $\mu(T)$ to satisfy $\mu=\mu_{0}$ and $\mathrm{d} \mu / \mathrm{d} T=-\lambda$ at $T=T_{0}$, where $\lambda$ is a positive constant.

We will consider only thin films, whose aspect ratio $\epsilon$ (defined by $\epsilon=\left(\mu_{0} U_{0} / \rho g a^{2}\right)^{1 / 2}$, where $U_{0}$ is a characteristic velocity defined by $U_{0}=\left(\rho g Q^{2} / \mu_{0}\right)^{1 / 3}$ for Nusselt flow and $U_{0}=a \Omega$ for Moffatt flow) satisfies $\epsilon \ll 1$. We scale the system by writing

$$
\left.\begin{array}{r}
r=a\left(1 \pm \epsilon y^{*}\right), \quad h=\epsilon a h^{*}, \quad u=U_{0} u^{*}, \quad v=\epsilon U_{0} v^{*}, \quad p-p_{\mathrm{a}}= \pm \epsilon \rho g a p^{*}, \\
Q=\epsilon U_{0} a Q^{*}, \quad M=\epsilon \rho a^{2} M^{*}, \quad T=T_{\infty}+\left(T_{0}-T_{\infty}\right) T^{*}, \quad \mu=\mu_{0} \mu^{*},
\end{array}\right\}
$$

where $Q$ is the azimuthal volume flux of fluid per unit axial length crossing a station $\theta=$ constant, $M$ is the mass per unit axial length of fluid on the cylinder, $p_{\mathrm{a}}$ is atmospheric pressure, $g$ is gravitational acceleration, and we have written $\mathbf{u}=u \mathbf{e}_{\theta}+v \mathbf{e}_{y}$, where $\mathbf{e}_{\theta}$ and $\mathbf{e}_{y}$ denote unit vectors in the directions of increasing $\theta$ and $y$, respectively; moreover, in the scaling for $r$ and $p$ the + sign refers to Nusselt flow or coating flow, and the - sign to rimming flow, so that in this representation the fluid occupies $0 \leq y^{*} \leq h^{*}(\theta)$ for $-\pi<\theta \leq \pi$. For clarity the star superscripts on non-dimensional variables will be dropped from now on.

At leading order in $\epsilon$ the governing equations give

$$
u_{\theta}+v_{y}=0, \quad\left(\mu u_{y}\right)_{y}=\cos \theta, \quad p_{y}=-\sin \theta, \quad T_{y y}=0,
$$

together with the boundary conditions

$$
u=U, \quad v=0 \quad \text { and } \quad T=1 \quad \text { on } \quad y=0
$$

(where $U=0$ for Nusselt flow and $U=1$ for Moffatt flow) and

$$
u_{y}=0, \quad p=0 \text { and } T=0 \text { on } y=h .
$$


Therefore the solution for $u, p$ and $T$ is

$$
u=U-h^{2} \cos \theta \int_{T}^{1} \frac{\tilde{T}}{\mu(\tilde{T})} \mathrm{d} \tilde{T}, \quad p=\sin \theta(h-y), \quad T=1-\frac{y}{h} .
$$

The mass $M$ of fluid per unit axial length is given by

$$
M=\int_{-\pi}^{\pi} h \mathrm{~d} \theta
$$

and the azimuthal volume flux of fluid per unit axial length is

$$
Q=\int_{0}^{h} u \mathrm{~d} y=U h-\frac{\cos \theta}{3} f h^{3}
$$

where $f=f(h)(>0)$ is the fluidity of the fluid film, defined by

$$
f=\frac{3}{h^{3}} \int_{0}^{h} \int_{0}^{y} \frac{h-\tilde{y}}{\mu(T(\theta, \tilde{y}))} \mathrm{d} \tilde{y} \mathrm{~d} y=\frac{3}{h^{3}} \int_{0}^{h} \frac{(h-y)^{2}}{\mu(T(\theta, y))} \mathrm{d} y .
$$

With $\mu(T)$ prescribed, the film thickness $h=h(\theta)$ is determined in terms of the flux $Q$ by the algebraic equation (7). Since the flow is steady, $Q$ is constant in Moffatt flow and is piecewise constant in Nusselt flow; moreover, in Nusselt flow $Q$ is prescribed, and so (6) serves only to determine the mass $M$ (if desired), whereas in Moffatt flow it is $M$ that is prescribed, and (6) must be included in order for the value of $Q$ (and hence the form of $h$ ) to be determined. Once $h$ is known the solution for $u, p$ and $T$ is given explicitly by (5). The fact that $h, u$ and $T$ depend on $\theta$ only through $\cos \theta$ shows that the flow has top-to-bottom symmetry, at least for the simple type of solution considered here. Putting $\theta=\pi$ in (7) we note that $Q>0$ in Moffatt flow; however, in Nusselt flow we have $Q>0$ on the left-hand side of the cylinder (i.e. $\frac{1}{2} \pi<|\theta| \leq \pi$ ), and $Q<0$ on the right-hand side (i.e. $|\theta|<\frac{1}{2} \pi$ ).

\subsection{Isothermal flow}

The fluidity $f$ is defined in such a way that if the cylinder is at the same temperature as the atmosphere, so that the fluid film is isothermal $\left(T=T_{0}=T_{\infty}\right)$ and the viscosity is constant $(\mu \equiv 1)$, then $f=1$. In that case we recover the isothermal problems in which $h=h_{0}, u=u_{0}$, $Q=Q_{0}$ and $M=M_{0}$, where

$$
u_{0}=U-\frac{\cos \theta}{2}\left(2 h_{0}-y\right) y, \quad Q_{0}=U h_{0}-\frac{\cos \theta}{3} h_{0}^{3}, \quad M_{0}=\int_{-\pi}^{\pi} h_{0} \mathrm{~d} \theta .
$$

In isothermal Nusselt flow $(U=0)$ we recover Nusselt's $[2,3]$ solution for $h_{0}$, namely

$$
h_{0}=\left(-\frac{3 Q_{0}}{\cos \theta}\right)^{1 / 3}
$$

representing a film of fluid incident on the cylinder from above, flowing round the cylinder, and falling off at the bottom. For isothermal Moffatt flow $(U=1)$ Moffatt [4] showed that $(9 \mathrm{~b})$ has a continuous solution $h$ provided that $M$ satisfies $0<M_{0} \leq M_{0 \mathrm{c}} \simeq 4.44272$ (and correspondingly $\left.0<Q_{0} \leq Q_{0 \mathrm{c}}=\frac{2}{3}\right)$, showing that, for example, in coating flow a continuous fluid film can be supported steadily on the cylinder against gravity by rotation of the cylinder only if its mass does not exceed the critical value $M_{0 \mathrm{c}}$. Johnson [5] further showed that discontinuous solutions that involve jumps ("shocks") in the free surface are also possible; these solutions, which are the only possible steady two-dimensional solutions when $M>M_{0 \mathrm{c}}$, are particularly relevant for rimming flow. 


\subsection{Non-isothermal flow}

In the present non-isothermal problem with large Biot number, equation (8) gives

$$
f=3 \int_{0}^{1} \frac{T^{2}}{\mu(T)} \mathrm{d} T,
$$

which shows immediately that in this case $f$ is a constant, independent of $\theta$. Therefore with the re-scaling

$$
h=\frac{h_{0}}{f^{1 / 2}}, \quad Q=\frac{Q_{0}}{f^{1 / 2}}, \quad M=\frac{M_{0}}{f^{1 / 2}}
$$

we recover from (5)-(7) the classical isothermal problem (9) for the film thickness $h$. Thus our rather surprising conclusion is that the solution $h$ for the non-isothermal Nusselt or Moffatt flow may be obtained from that of the corresponding isothermal problem merely by a simple re-scaling. In particular, in Moffatt flow the critical mass is $M_{\mathrm{c}}=M_{0 \mathrm{c}} f^{-1 / 2} \simeq 4.44272 f^{-1 / 2}$, occurring when $Q=Q_{0 \text { c }} f^{-1 / 2}=\frac{2}{3} f^{-1 / 2}$. On the other hand, from (5) we note that the velocity profile in non-isothermal cases is quite different from the semi-parabolic profile in (9) in the isothermal case (and the streamline pattern is correspondingly different). For example, for an exponential viscosity model given (in dimensional terms) by

$$
\mu(T)=\mu_{0} \exp \left(-\frac{\lambda\left(T-T_{0}\right)}{\mu_{0}}\right)
$$

we have $\mu=\exp (V y / h)$, where $V$ is the non-dimensional thermoviscosity number defined by $V=\lambda\left(T_{0}-T_{\infty}\right) / \mu_{0}$, and the velocity $u$ and fluidity $f$ are found to be

$$
u=U-\frac{h \cos \theta}{V^{2}}\left[(V-1) h-(V h-V y-h) \exp \left(-\frac{V y}{h}\right)\right]
$$

and

$$
f=\frac{3}{V^{3}}\left[(V-1)^{2}+1-2 \exp (-V)\right] .
$$

A related conclusion was arrived at by Wilson and Duffy [6] in their study of the unsteady gravity-driven draining of a thin rivulet of fluid with temperature-dependent viscosity down a heated or cooled substrate; they showed that in the limit of large Biot number the evolution equation for such a rivulet can be obtained directly from the corresponding equation for an isothermal rivulet by re-scaling time and flux with the fluidity $f$ (and therefore that in steady flow only the flux need be re-scaled).

\section{Conclusions}

We investigated two-dimensional steady flow of a thin film of fluid with temperature-dependent viscosity on a uniformly heated or cooled horizontal cylinder, which may be stationary or rotating uniformly about its axis, in the case when the Biot number is large. We showed that the solution for the fluid velocity, pressure and temperature is expressible in terms of the film thickness $h$, and we derived the algebraic equation (7) that determines $h$. We showed that the film thickness (but not the fluid velocity) can be obtained from that in the isothermal case by a simple re-scaling.

\section{References}

1. S. F. Kistler, P. M. Schweizer (editors), Liquid Film Coating (Chapman \& Hall, 1997)

2. W. Nusselt, Z. Vereines deutscher Ingenieure 60, 541-546 (1916)

3. W. Nusselt, Z. Vereines deutscher Ingenieure 60, 569-575 (1916)

4. H. K. Moffatt, J. Méc. 16, 651-673 (1977)

5. R. E. Johnson, J. Fluid Mech. 190, 321-342 (1988)

6. S. K. Wilson, B. R. Duffy, J. Engng Maths 42, 359-372 (2002) 